\title{
Study on neutron scattering in light water
}

\author{
Juan Pablo Scotta ${ }^{1 a}$, Ignacio Marquez Damian ${ }^{2}$, Gilles Noguere ${ }^{1}$, and David Bernard ${ }^{1}$ \\ ${ }^{1}$ SPRC/LEPh, CEA Cadarache, F-13108 Saint-Paul-Lez-Durance, France \\ ${ }^{2}$ Neutron Physics Department, Centro Atomico Bariloche, Argentina
}

\begin{abstract}
It is presented a method to produce covariance matrices of the light water total cross section from thermal scattering laws of the JEFF-3.1.1 nuclear data library and CAB model. The generalized least square method was used to fit the LEAPR module parameters of the processing tool NJOY with light water experimental transmission measurements at $293.6 \mathrm{~K}$ with CONRAD code. The marginalization technique was applied to account for systematic uncertainties.
\end{abstract}

\section{Introduction}

The probability of an incident neutron with energy and solid angle $(E ; \Omega)$ of scattering a light water molecule and being emitted at a different energy and different solid angle $\left(E^{\prime} ; \Omega^{\prime}\right)$ can be expressed with the double differential scattering cross section:

$$
\frac{d^{2} \sigma}{d \Omega d E}\left(E \rightarrow E^{\prime}, \Omega \rightarrow \Omega^{\prime}\right)=\frac{\sigma_{b}}{4 \pi k T} \sqrt{\frac{E^{\prime}}{E}} e^{-\frac{\beta}{2}} S(\alpha, \beta)
$$

where $\sigma_{\mathrm{b}}$ is the characteristic bound cross section of the material, $\mathrm{T}$ is the temperature and $\mathrm{k}$ is the Boltzmann constant.

The double differential cross section depends on the thermal scattering law function $S(\alpha, \beta)$, where $\alpha$ and $\beta$ are the dimensionless momentum and energy transfer respectively:

$$
\alpha=\frac{E^{\prime}+E-2 \sqrt{E^{\prime} E} \cos \theta}{A k T} ; \beta=\frac{E^{\prime}-E}{k T}
$$

where $\cos \theta$ is the cosine of the scattering angle in the laboratory system and $A$ is the ratio between the mass of the scattering molecule and the neutron mass.

\footnotetext{
${ }^{\mathrm{a}}$ Corresponding author: juan-pablo.scotta@cea.fr
} 
In order to evaluate the function $S(\alpha, \beta)$ some approximations are often used: the incoherent and the Gaussian approximation. The processing of the thermal scattering law is done with LEAPR module of NJOY code [1], and is computed with these approximations.

The main parameter that defines the dynamics of the function $S(\alpha, \beta)$ is the frequency spectrum of the material, which defines its excitation states. When using LEAPR module, the frequency spectrum is a resultant of three different components: a solid type spectrum, a translational spectrum and discrete oscillators.

\section{Models for $\mathrm{H}$ into $\mathrm{H} 2 \mathrm{O}$}

It will be presented two different models that describe the frequency distribution of $\mathrm{H}$ into $\mathrm{H}_{2} \mathrm{O}$.

The first one is IKE model, developed by M. Mattes and J. Keinert [2]. It is based on experimental frequency spectrum measured by B. C. Haywood and D. I. Page [3]. The rotational mode of hydrogen bound to the water molecule is represented by a solid type spectrum with a weight $\mathrm{w}_{\mathrm{R}}$. Two discrete oscillators, with energies $E_{1}$ and $E_{2}$, define the intra-molecular modes of vibration (bending and stretching modes), with corresponding weight $\mathrm{w}_{1}$ and $\mathrm{w}_{2}$. Finally, an effective mass describes the molecular clustering effect of the water molecule with a weight $\mathrm{w}_{\mathrm{t}}$. The sum of all the weights must be equal to unity.

The nuclear data library JEFF-3.1.1 adopted this model in its database. Table 1 resumes the LEAPR parameters of this model at $293.6 \mathrm{~K}$.

The second model, CAB model, was calculated by I. Marquez Damian [4] and is based on molecular dynamic simulations with GROMACS code. It was obtained an equivalent model to IKE model but the translational mode was replaced by the diffusional model of Egelstaff-Schofield [5] to better describe low energy dynamics. The key parameter in this model is the diffusion constant c. Table 1 resumes the LEAPR parameters of CAB model at 293.6K.

Figure 1 compares the frequency spectrum for IKE model (used in the JEFF-3.1.1 nuclear data library) and CAB model at 293.6K.

The total cross section is obtained integrating equation (1) in all emitted neutron energies and solid angles. It was computed for JEFF-3.1.1 library and CAB model at 293.6 K. Figure 2 compares them with experimental data [6-8].

Table 1. IKE and CAB model parameters of $\mathrm{H}$ into $\mathrm{H} 2 \mathrm{O}$ at $293.6 \mathrm{~K}$ for the LEAPR module of NJOY.

\begin{tabular}{|l|c|c|}
\hline \multicolumn{1}{|c|}{ Frequency spectrum parameters } & JEFF - 3.1.1 & CAB Model \\
\hline Translational weight $\boldsymbol{\omega}_{\mathrm{t}}$ & 0.021739 & 0.007918 \\
\hline Solid-type spectrum weight $\boldsymbol{\omega}_{\mathrm{R}}$ & 0.489130 & 0.522080 \\
\hline Scissoring mode oscillator energy $\mathbf{E}_{2}(\mathrm{meV})$ & 205 & 205 \\
\hline Scissoring mode oscillator weight $\boldsymbol{\omega}_{2}$ & 0.16304 & 0.15667 \\
\hline Stretching modes oscillator energies $\mathbf{E}_{1,3}(\mathrm{meV})$ & 436 & 430 \\
\hline Stretching modes oscillator weights $\boldsymbol{\omega}_{1,3}$ & 0.3261 & 0.31333 \\
\hline Dimensionless diffusion constant $\mathbf{c}$ & 0 & 3.969 \\
\hline
\end{tabular}




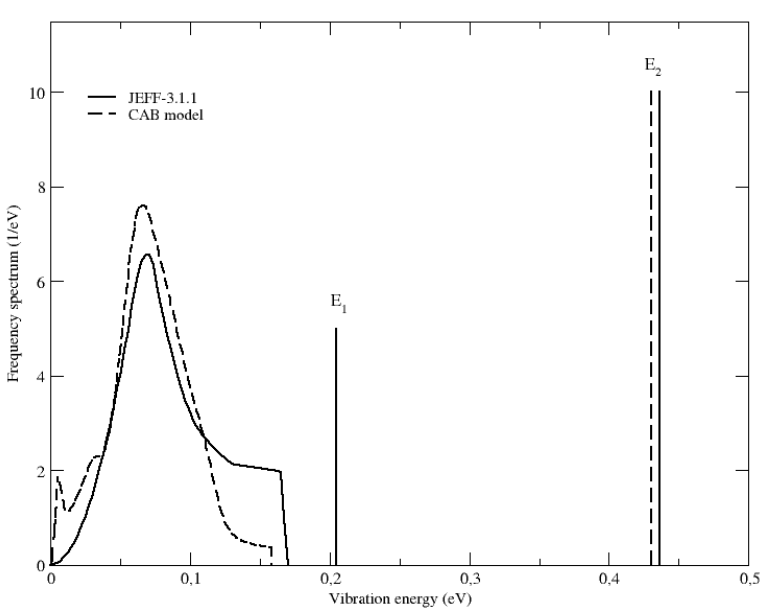

Figure. 1. Frequency spectrum for JEFF-3.1.1 and CAB model at 293.6 K.

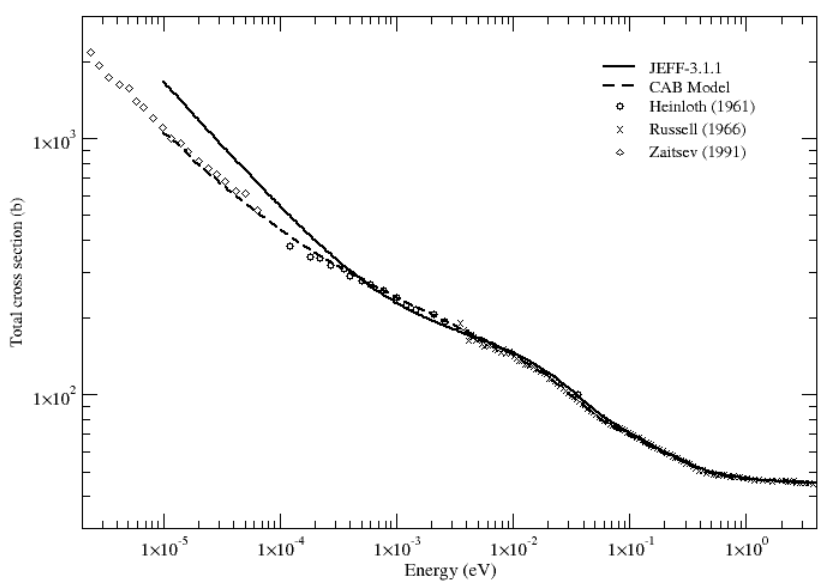

Figure. 2. Total cross section for JEFF-3.1.1 and CAB model, at 293.6 K.

\section{Covariance matrices of differential and total cross section}

\subsection{Governing equations}

In this work, the full covariance matrix between the model parameters is defined as follow:

$$
\Sigma=\left(\begin{array}{ll}
\Sigma_{11} & \Sigma_{12} \\
\Sigma_{21} & \Sigma_{22}
\end{array}\right)
$$

where each element of the matrix is obtained by:

$$
\begin{aligned}
& \Sigma_{11}=M_{x}+\left(G_{x}^{T} G_{x}\right)^{-1} G_{x}^{T} G_{\theta} M_{\theta} G_{\theta}{ }^{T} G_{x}\left(G_{x}^{T} G_{x}\right)^{-1} \\
& \Sigma_{12}=-\left(G_{x}^{T} G_{x}\right)^{-1} G_{x}^{T} G_{\theta} M_{\theta}
\end{aligned}
$$




$$
\Sigma_{22}=M_{\theta}
$$

in which $\mathrm{M}_{\mathrm{x}}$ stands for the covariance matrix between the best-fit values of the model parameter and $\mathrm{M}_{\theta}$ represents the covariance matrix between the nuisance parameters that account systematic uncertainties. The nuisance parameters included in the present study were the normalization and the background. In our context, the model parameter vector $\mathrm{x}$ and the nuisance parameter vector $\theta$ are:

$$
\overrightarrow{\mathrm{x}}=\left(c, \omega_{t}, \omega_{R}, \Delta, E_{1}, E_{2}, \omega_{1}, \omega_{2}\right) ; \vec{\theta}=(N, B)
$$

The matrices $G_{x}$ and $G_{\theta}$ correspond to the derivatives of the calculated quantity $z$ to the model and the nuisance parameters respectively:

$$
G_{x}=\left(\begin{array}{cccc}
\frac{\partial z_{1}}{\partial c} & \frac{\partial z_{1}}{\partial \omega_{t}} & \ldots & \frac{\partial z_{1}}{\partial \omega_{2}} \\
\frac{\partial z_{2}}{\partial c} & \frac{\partial z_{2}}{\partial \omega_{t}} & \ldots & \frac{\partial z_{2}}{\partial \omega_{2}} \\
\vdots & \vdots & \ldots & \vdots \\
\frac{\partial z_{k}}{\partial c} & \frac{\partial z_{k}}{\partial \omega_{t}} & \ldots & \frac{\partial z_{k}}{\partial \omega_{2}}
\end{array}\right) ; G_{\theta}=\left(\begin{array}{cc}
\frac{\partial z_{1}}{\partial N} & \frac{\partial z_{1}}{\partial B} \\
\frac{\partial z_{2}}{\partial N} & \frac{\partial z_{2}}{\partial B} \\
\vdots & \vdots \\
\frac{\partial z_{k}}{\partial N} & \frac{\partial z_{k}}{\partial B}
\end{array}\right)
$$

To calculate the covariance matrices of the total cross sections CONRAD code [9] was used. The Generalized Least Square method (GLS) was used in association with the marginalization technique in which the zero variance penalty condition accounts for systematic uncertainties [10].

The "best estimates values" of the LEAPR module parameters were obtained from water transmission experimental measures.

\subsection{Sensitivity analysis of the model parameters}

A sensitivity analysis of the LEAPR module parameters to the $\mathrm{H}_{2} \mathrm{O}$ transmission at $293.6 \mathrm{~K}$ was done. Their sensitivity as a function of the incident neutron energy is plotted in figure 3 for the $\mathrm{H}_{2} \mathrm{O}$ transmission calculated with $\mathrm{CAB}$ model.

The parameters $\omega_{\mathrm{R}}$ and $\Delta$ are one order of magnitude more sensitive to the transmission than the rest. This highlights the relevance of the continuous part of the frequency spectrum, described by these two parameters. Their anticorrelation suggests that only one of them should be taken into account in the marginalization technique.

The parameters $\mathrm{c}$ and $\omega_{\mathrm{t}}$ follow an equivalent trend because they are physically correlated. The parameters not included in figure $3\left(\mathrm{E}_{1}, \mathrm{E}_{2}, \omega_{1}\right.$ and $\left.\omega_{2}\right)$ have a negligible sensitivity. Their uncertainties are not taken into account in the present study. 


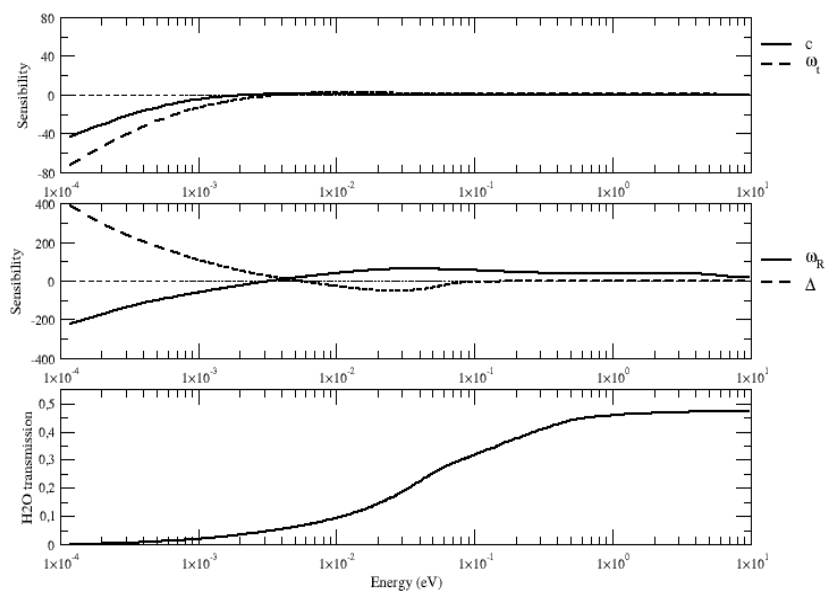

Figure. 3. Sensitivity (in \%/\%) of the LEAPR model parameters to the $\mathrm{H} 2 \mathrm{O}$ transmission as a function of the energy calculated with $\mathrm{CAB}$ model.

\subsection{Results}

According to our sensitivity analysis, the uncertainty on the total cross section is mainly driven by the parameters $\Delta$ and $\omega_{t}$. The relative uncertainties and the correlation matrices of the total cross section for JEFF-3.1.1 and CAB model at 293.6 $\mathrm{K}$ are shown in figure 4.

In the cold neutron energy range, below $10^{-3} \mathrm{eV}$, the uncertainty associated to JEFF-3.1.1 is larger than the uncertainty associated to $\mathrm{CAB}$. This is explained by the better agreement of the CAB model with the low energy total cross section measurements.

Around $5 \mathrm{meV}$, the uncertainty found for $\mathrm{CAB}$ model is low. Additional work is needed for improving this underestimated magnitude.

Above the thermal energy, the relative uncertainties remain lower than $2 \%$. The main contribution is related to the ${ }^{1} \mathrm{H}$ free atom cross section whose uncertainty is lower than $1 \%$ [11].

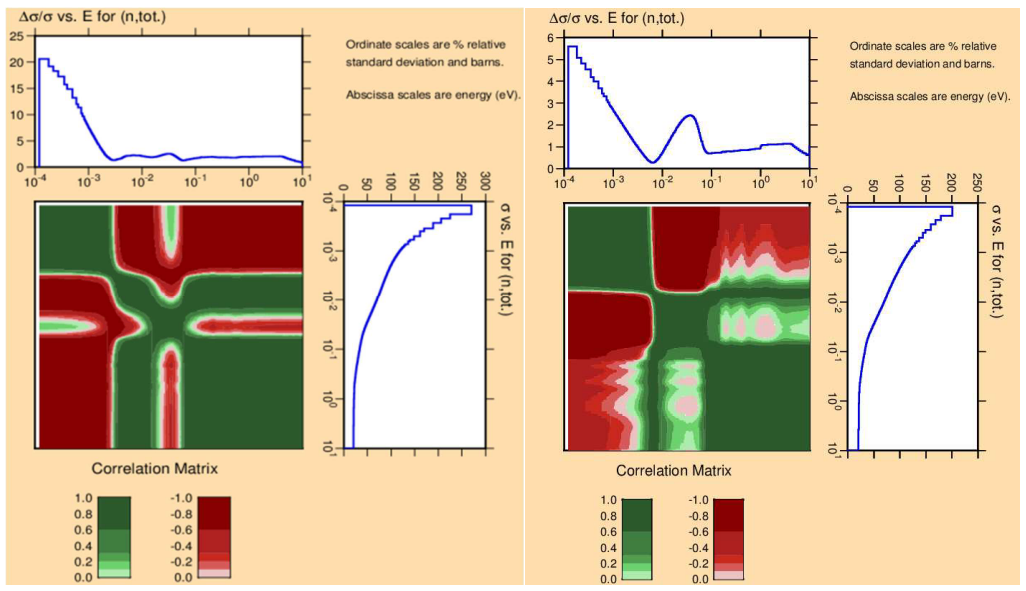

Figure. 4. Relative uncertainties and correlation matrices of the total cross section for JEFF-3.1.1 (left) and CAB model (right) at $293.6 \mathrm{~K}$. 


\section{Conclusions}

The covariance matrices of the light water total cross sections from thermal scattering laws of JEFF3.1.1 nuclear data library and $\mathrm{CAB}$ model were obtained by using CONRAD code. In the cold neutron energy range, differences in the relative uncertainties between both models are important. The uncertainties associated to JEFF-3.1.1 are larger because the agreement with the low energy total cross section is poor.

Present work is being done to propagate uncertainties due to the thermal scattering laws to integral calculations. Preliminary calculations performed on MOX fuel provide uncertainties on the multiplication factor ranging from $100 \mathrm{pcm}$ with the CAB model to $200 \mathrm{pcm}$ with JEFF-3.1.1.

The next step would be to determine uncertainties on the water potential parameters used in the molecular dynamic simulation code GROMACS, in order to obtained directly uncertainties on the frequency spectrum introduced in LEAPR module.

\section{References}

1. R.E. MacFarlane and A.C. Kahler, Nucl. Data Sheets 111, 2729 (2010).

2. M. Mattes and J. Keinert, "Thermal Neutron Scattering Data for the moderator Materials H2O, D2O and ZrHx in ENDF-6 Format and as ACE Library for MCNP(x) Codes", International Atomic Energy Agency, INDC(NDS)-0470, 2005.

3. D.I. Page, B.C. Haywood, "The Harwell scattering law program: frequency distributions of moderators", Harwell Report AERE-R 5778, 1968.

4. J. I. Marquez Damian et al, Ann. Nucl. Ener. 65, 280 (2014).

5. P.A. Egelstaff and P. Schofield, Nucl. Sci. Eng. 12, 260 (1962).

6. J.L. Russell Jr., J.M. Neill and J.R. Brown, "Total cross section measurements of H2O", General Atomic Report GA-7581, 1966.

7. K.N. Zaitsev, V.N. Petrov, S.P. Kuztnesov, O.A. Langer, I.V. Meshkov, A.D. Perekrestenko, Sov. At. Energy 70, 238 (1991).

8. K. Heinloth, Z. Phys. 163, 218 (1961).

9. C. De Saint Jean et al., "CONRAD, a new software for nuclear data analysis", Nuclear Energy Agency, JEFFDOC-1142, 2006.

10. G. Noguere, P. Archier and C. De Saint Jean, Nucl. Sci. Eng. 172, 164 (2012).

11. S. Mughabghab, "Atlas of Neutron Resonance", Elsevier, 2006. 